\title{
PRASSI SCRITTORIA NEL XIV SECOLO: LINGUA E CULTURA NEL GIUDICATO SARDO DI ARBOREA ${ }^{1}$
}

I documenti del giudicato di Arborea costituiscono una fonte preziosa per lo studio della complessa situazione linguistica in Sardegna. La varietà sarda parlata nell'area arborense, come è noto, è considerata ormai un sistema autonomo rispetto alle altre varietà rappresentate in particolar modo dal campidanese e dal logudorese. Caratterizzato da tratti alcuni dei quali sono condivisi dal campidanese mentre altri sono tipici del logudorese, l'arborense è stato definito per lungo tempo come una varietà "mista", tipica delle aree di confine, delle zone grigie, influenzata dai due dialetti sardi contigui che sono appunto il campidanese e il logudorese.

Nell'indagare la lingua della Carta de Logu, uno dei più importanti documenti del giudicato di Arborea, Guarnerio (1905) sottolinea la caratteristica "mista" della parlata arborense dovuta al fatto che l'area costituisce il punto nel quale vengono in contatto il logudorese e il campidanese; la diffusione del campidanese nella zona di Oristano e il mantenimento di fenomeni tipici del logudorese, con l'innovazione di altri tratti sempre del logudorese, avrebbero contribuito a rendere sempre più composito il carattere dell'arborense. Al contrario Sanna (1975) rileva la specificità dell'arborense che non può essere considerato come la parlata di una zona grigia, un miscuglio di campidanese e di logudorese, bensì come una varietà dotata di una propria autonomia che si contrappone agli altri dialetti sardi. Lo studioso sottolinea soprattutto talune peculiarità dell'arborense rappresentate dall'oscillazione nelle terminazioni in - $e$ e in - $o$ e dall'alternanza di -os e di - $u s$ per la formazione del plurale; queste terminazioni caratterizzano i sistemi linguistici contigui, dal momento che $-e$ ed -os sono tipici del logudorese, mentre - $i$ ed - us sono peculiari del campidanese; tuttavia queste alternanze caratterizzano anche la fase arcaica del campidanese, come è possibile constatare nelle antiche carte campidanesi (le Carte Volgari dell'Archivio Arcivescovile di Cagliari pubblicate dal Solmi 1905, la Carta cagliaritana in caratteri greci a proposito della quale vd. Blancard-Wescher 1874). Secondo Sanna quindi talune caratteristiche dell'arborense, che sono state considerate dagli studiosi come tratti peculiari del logudorese contrapposti a quelli campidanesi, compaiono nelle due maggiori varietà sarde nella fase arcaica, prima che intervenisse il processo di diversificazione tra il logudorese e il campidanese che in quest'ultima varietà ha condizionato il cambiamento delle terminazioni $-e$ ed $-o s$ rispettivamente in $-i$ ed $-u s$. L'arborense si presenta pertanto come una varietà autonoma "con caratteri antichi e

$1 \quad$ La ricerca è stata effettuata con un contributo del M.U.R.S.T. 
nuovi che si incontrano: per intenderci, caratteri di tipo logudorese (che non sono però imitazioni logudoresi, ma forme genuine) e innovazioni campidanesi, in un'area intermedia, come ci dimostrano chiaramente i dialetti odierni di questa zona" (Sanna 1975: 124).

Questi tratti sono rilevabili nelle antiche carte arborensi, ovvero nella carta del XII secolo concernente la conferma da parte di Orzoccor de Zori dell'affrancamento delle ville di Nuraghe Nigellu e di Masone de Capras, deciso da donna Nibata, (cfr. Besta 1906: 423-33; Volpicella 1926: 63-90; Monteverdi 1935: 34-6; Lazzeri 1942: 58-66) e nella carta del 1102 relativa ad una permuta di beni tra il giudice Torbeno e Costantino d'Orrubu (cfr. Merci 1978). Oltre a queste carte, i documenti più importanti redatti nella varietà arborense sono rappresentati dal condaghe di Santa Maria di Bonarcado del XII secolo, dal Brogliaccio di San Martino di Oristano, dal Condaxi Cabrevadu, dal condaghe di Santa Chiara di Oristano e dalla Carta de Logu de Arborea.

I tratti che consentono la caratterizzazione dell'arborense rispetto alle principali varietà sarde sono stati indagati da Sanna (1975: 121-68) e recentemente da Virdis (1982 e 1988) e da Maninchedda (1987 e 1987 a). Mentre rinviamo soprattutto a Virdis (1988) per una esauriente analisi delle principali caratteristiche dell'arborense, ci soffermiamo su un aspetto linguistico dell'area arborense, rappresentato dall'interferenza rilevabile negli antichi documenti e derivante dalle altre lingue (il toscano e il catalano) che, per le note vicende storiche che hanno riguardato la Sardegna e in particolar modo il giudicato di Arborea ${ }^{2}$, hanno influenzato la parlata locale e la prassi scrittoria. Come vedremo, la presenza di altri sistemi non favorisce solamente il fenomeno dell'interferenza; dall'epistolario dei regoli del giudicato di Arborea, per esempio, si ricavano suggestive informazioni sul plurilinguismo che doveva caratterizzare il giudicato e si nota l'alternanza dei codici nella stesura delle lettere da parte dei regoli e dei loro familiari.

L'influenza del toscano $\mathrm{e}$, in un periodo più tardo, del catalano e dello spagnolo nei documenti arborensi testimonia lo stretto contatto che si manifesta fra la cultura locale e quella dei dominatori, ma non solo dei dominatori poiché il toscano è impiegato nella prassi scrittoria anche in un periodo in cui la Sardegna faceva parte ormai del regno di Aragona. Merci (1978: 365) sottolinea l'occorrenza del toscano mezetima "mercoledi" nella carta del 1102, esito che erroneamente è stato interpretato "giovedì" dagli studiosi precedenti; come rileva Merci (1978: 376) la presenza di questa forma toscana nell'area sarda è stata segnalata da Wagner (1951 e DES), ma è attestata solo da questa carta. Altri toscanismi sono attestati invece da altre carte; le forme toscane del condaghe di S. Maria di Bonarcado ancu "anche", preiti "prete" ricorrono rispettivamente nelle Carte Volgari dell'Archivio Arcivescovile di Cagliari, negli Statuti Sassaresi, negli Statuti di Castelsardo e nella Carta de Logu (ancu), e nei condaghi di San Pietro di Silki e di San Nicola di Trullas, nelle Carte Volgari cagliaritane (preiti) ${ }^{3}$.

2 Cfr. Artizzu (1973 e 1985), Casula (1978 e 1979).

3 Segnaliamo anche l'occorrenza nello stesso condaghe del toscanismo mustarolu "tipo di panno"; per 
Sanna (1975: 167) rileva alcuni esiti del condaghe che possono essere considerati dei toscanismi: si tratta delle forme sincopate quattru e atru (=antico toscano atro; cfr. Wagner DES, s.v. atteru) che si contrappongono alle forme logudoresi battoro e atteru.

Altri testi arborensi, appartenenti al XVI secolo, sono il Brogliaccio del convento di San Martino di Oristano, edito da Atzori (1956), e il Condaxi Cabrevadu, la cui edizione è stata curata ugualmente dall'Atzori (1957); questi testi, come afferma Sanna (1975: 158), costituiscono un'interessante fonte per lo studio del'evoluzione subita dalla varietà arborense $e$, per quel che concerne la nostra indagine, rappresentano una testimonianza dell'influenza esercitata dalle altre lingue con le quali l'arborense è in contatto, il toscano e lo spagnolo. Nel Brogliaccio di S. Martino Sanna (1975: 160-1) rileva la presenza di toscanismi (per esempio voltas, considerato dallo studioso come un prestito non assimilato) e di esiti fonetici che riproducono il modello toscano (per esempio $r j>i$ in nodaiu, oberaiu), mentre a proposito della lingua del Condaxi $\mathrm{Ca}$ brevadu si osserva che "Trattandosi di linguaggio e di formulario notarile abbonda l'influsso culto latino. Frequenti gli influssi lessicali spagnoli: più numerosi quelli toscani" (Sanna 1975: 163).

Il condaghe di Santa Chiara di Oristano, la cui edizione è curata da Maninchedda (1987), conferma l'influsso toscano nella prassi scrittoria dell' area arborense; questo condaghe, che risale agli anni tra la fine del Quattrocento e gli inizi del Cinquecento come afferma il curatore, manifesta una notevole infuenza proveniente dagli ambienti culturali della penisola italiana ed è una fonte preziosa per lo studio delle componenti linguistiche che interagiscono nell' isola. Redatto prevalentemente nella varietà sarda arborense, il condaghe contiene testi scritti in catalano; il procuratore del convento Bartolo Passiu che ha redatto la maggior parte dei contratti "scriveva in sardo mentre gli altri procuratori scrivevano prevalentemente in catalano; alcuni sono bilingui" (Maninchedda 1987 a: 366). Nei contratti redatti in sardo Maninchedda (1987 e 1987 a) rileva l'influsso del toscano e del catalano; per l'influenza della lingua iberica si vedano per esempio buida, che risente della forma catalana vuyt, mongas dal cat. monja, complidos in annos passados e complidos (cfr. la forma cat. coplit, Maninchedda 1987: 20).

Nell'ambito dell'influsso esercitato dalle lingue iberiche Maninchedda (1987 a) rileva l'occorrenza di alcuni esiti influenzati dallo spagnolo, quali patiu < patio, costadu a proposito del quale il Wagner (DES, s.v. kòsta) afferma "probm. = spagn. costado, cat. = costat", e infine calong $u$ che "potrebbe non essere l'esito della caduta della postonica di CANONICUS, ma dell'influsso dello spagn. calonge agevolato dalla dissimilazione consonantica $n-n>l-n$ tipica del campidanese" (Maninchedda 1987 a: 370).

L'influenza del toscano è certamente più sensibile e può essere rilevata non solo a livello lessicale, ma anche a livello morfo-sintattico: la terminazione $-o$ al posto di

le forme toscane nel condaghe di S. Maria di Bonarcado cfr. Wagner (1951:250 e 255). 
- $u$ in numerosi esiti lessicali, l'occorrenza di sequenze quali sa suprascrita, sa propria manu, su qualli ${ }^{4}$ testimoniano una certa incidenza del toscano; ma è soprattutto a livello lessicale che ne notiamo l'influsso come confermano i numerosi toscanismi forniti da Maninchedda (1987 e 1987 a), molti dei quali non sono registrati dal Wagner: si vedano gli esiti lessicali largamenti, pezu, protanti, confinis, medianti, quarteri, dimmodo, apartamentus, ecc. ${ }^{5}$

Il condaghe di S. Chiara costituisce un interessante documento per l'esame non solo dell'interferenza linguistica, ma anche dell'alternanza dei codici nelle carte redatte da una stessa persona: le carte 10 v., 53 v., 37 r. scritte da Perot Porrita sono redatte in catalano e in sardo, e ugualmente le carte $82 \mathrm{v}$. e 83 v., il cui scrivente è ignoto, sono redatte rispettivamente in sardo e in catalano. "Il condaghe di S. Chiara rappresenta, dunqe, il chiaro esito di un confronto tra tre codici linguistici: il sardo, il catalano e l'italiano" (Maninchedda 1987: 19).

Fra i documenti dell'area arborense occupa un posto privilegiato la Carta de Logu, un documento di particolare importanza per gli studi giuridici e per la conoscenza delle antiche consuetudini del giudicato. La Carta, promulgata da Eleonora di Arborea nel 1392, è un'opera composita, il cui nucleo originario è costituito probabilmente dalle leggi del regolo Mariano IV; ma allo stato attuale delle ricerche non è possibile operare una distinzione fra le disposizioni attribuibili al giudice e quelle dovute alla figlia Eleonora. Della Carta de Logu de Arborea possediamo un unico manoscritto, quello cagliaritano, e disponiamo di numerose edizioni, non tutte affidabili; l'edizione Besta-Guarnerio (1905) rimane l'unica stampa che presenti una relativa correttezza nella lettura del manoscritto, mentre l'edizione di Mameli (1805), pur corredata di un'utile traduzione in italiano, risente degli errori delle stampe precedenti delle quali il Mameli si serve per la sua edizione.

Nello studio sulla lingua della Carta de Logu Sanna (1975: 121-40) rileva più volte i tratti linguistici peculiari del documento, appartenente ad un'area che fino al $\mathrm{XV}$ secolo non risente dell'influenza del catalano; infatti per quanto "riguarda l'area arborense non va dimenticato che, se vi erano penetrate le forme toscane, essa aveva opposto resistenza militare e politica, prima di tutto alla penetrazione aragonese. Il che significa che mentre l'influsso catalano era assai diffuso nel campidanese, esso fu assente quasi del tutto fino al XV secolo nell'area arborense. E [...] proprio la conservazione nella Carta di una forma come $e b b a$ (equa) rispetto all'innovazione aqua (dal toscano acqua) che sostituì l'esito sardo autentico abba dimostra questa mancata penetrazione del catalano. Egua infatti, già presente ai tempi della compilazione della Carta nel campidanese (ma non nella zona arborense) è forma catalana" (Sanna 1975: 126). Tanto il Guarnerio (1905) quanto il Sanna (1975) sottolineano la preponderanza dell'influsso toscano nella redazione della Carta. Sanna (1975: 136) manifesta dei dubbi circa la presenza dell'influsso iberico nella scrittura; forme lessicali del tipo

$4 \quad$ Gli esempi sono tratti da Maninchedda (1987: 20).

5 Un elenco completo è contenuto in Maninchedda (1987 a: 388). 
desviadu, mescladura, biage con le varianti biagio e biatgio potrebbero essere attribuite a cause differenti, senza escludere la possibilità della presenza di uno scrivano spagnolo nel giudicato di Arborea; gli esiti desviadu e mescladura potrebbero essere forme sarde, secondo Sanna, mentre l'occorrenza di biage può essere dovuta ad una scrittura o ad una lettura non attendibile. Ugualmente la resa grafica que-, gue-per che-, ghe-, che potrebbe essere considerata di tipo iberico, richiama la grafia genovese (cfr. Sanna 1975: 136). Una lingua composita, quindi, quella della Carta de Logu, nella quale certamente è presente un notevole influsso toscano (e su questo aspetto concordano il Guarnerio e il Sanna); nello stesso tempo però non possiamo escludere altri tratti esogeni.

Vogliamo soffermarci ora proprio sulla compresenza dei sistemi linguistici nell'area arborense, prendendo in esame non solo l'interferenza linguistica ma anche l'alternanza dei codici. L'uso delle diverse lingue da parte di un medesimo utente è significativo ai fini dell'approfondimento delle componenti linguistiche che interagiscono in una data area, sia perché quest'uso è una prova della familiarità che l'utente ha con le diverse lingue che fanno parte del proprio repertorio, sia perché l'impiego alternativo è la testimonianza della scelta consapevole di questa o quella lingua ai fini comunicativi, scelta che è condizionata da molteplici fattori; ma l'impiego alternativo dei codici è un'ulteriore testimonianza soprattutto della variegata composizione culturale della zona.

L'assetto linguistico diversificato dell'area arborense è riflesso negli antichi documenti, nei quali i prestiti potrebbero costituire solo una conferma della progressiva penetrazione delle culture esogene dovuta alle diverse dominazioni che si sono succedute in Sardegna. Come è noto, nell'isola si sono avute le dominazioni pisana e genovese a partire dall'XI secolo e fino al 1323, anno in cui la Sardegna entra a far parte della compagine catalana e in seguito, con l'unificazione dei regni di Aragona e di Castiglia (1479), di quella spagnola. In un periodo in cui la lingua dei dominatori è quella catalana o spagnola, i prestiti toscani potrebbero rappresentare delle forme penetrate precedentemente $\mathrm{e}$ che sono state ormai assimilate dalla varietà sarda; ma $\mathrm{i}$ documenti dell'area arborense testimoniano l'uso del toscano durante la dominazione catalana e spagnola.

Di particolare importanza a questo proposito sono i documenti della cancelleria del giudicato di Arborea, l'unico stato indigeno che sopravvive fino al 1410. Il giudicato di Arborea, a proposito del quale esaminiamo l'uso di diverse lingue negli scritti dei regoli e dei loro familiari, è un'area privilegiata per lo studio del plurilinguismo. La storia del giudicato di Arborea segue certamente le sorti dell'isola ${ }^{6}$; vediamo i giudici di Arborea allearsi con i pisani e i genovesi, a seconda degli eventi, durante la dominazione delle due repubbliche in Sardegna, così come assistiamo all'alleanza dei regoli sardi con i catalani a partire dal 1323 , anno in cui l'isola entra a far parte della

6 Per gli eventi storici della Sardegna a partire dall 'XI e fino al XIV secolo rinviamo ad Artizzu (1985) e ad Anatra (1984). 
compagine iberica, e fino al 1353 quando fra l'Arborea e i catalani scoppia la guerra che segna l'opposizione dei regoli nei confronti dei dominatori aragonesi. L'ultimo giudicato sardo, dopo la fine degli altri giudicati isolani avvenuta in seguito alla sottomissione della Sardegna alla Corona di Aragona, sopravviverà fino al 1410.

Nella prima fase della dominazione catalana, ovvero fino al 1353, i giudici condividono gli interessi aragonesi e, tramite un'abile politica matrimoniale, intensificano i rapporti con le più importanti casate iberiche. Come rileva Casula (1979: 75) il "giudice Ugone II (1321-1335) [...] fece sposare ben sei dei suoi dieci figli - escluso il primogenito e successore Pietro III (1335-1347) - con donzelli e donzelle di famiglie iberiche, dando origine a grandi e prestigiose casate spagnole, quali quelle dei Vilamarin, dei Medinaceli, dei Montalto, dei Soma"; lo stesso giudice preferisce che due dei suoi figli, Mariano e Giovanni, si formino alla corte aragonese dove soggiornano a lungo e dove ricevono un'adeguata istruzione. D'Arienzo (1968) ha messo in rilievo i profondi legami che sussistono fra gli ambienti catalani e i giudici arborensi, che nella penisola iberica hanno ricchi possedimenti comprendenti numerosi castelli e terre. Nonostante questi legami, la cultura del giudicato di Arborea risente dell'jnfluenza dei maggiori centri della nostra penisola.

Questa presenza dei moduli toscani o genovesi durante la dominazione catalana può essere intesa come il perdurare dei modelli importati dai dominatori precedenti, così come può essere considerata come un'accettazione volontaria di nuovi istituti al fine di affermare l'indipendenza rispetto al potere aragonese; tuttavia se "questa influenza fosse di natura nuova o tradizionale, se, cioè, vi fosse stato da parte degli Oristanesi un riavvicinamento alla penisola italiana nel periodo di guerra dell'Arborea, dal ' 53 in poi, o se il forte istinto conservativo dei Sardi si manifestasse ancora una volta nella conservazione dei modi e degli istituti italiani pre-aragonesi, inconsciamente, o magari per affermare di fronte agli Iberici la propria indipendenza e la propria sovranità, questo è ancora da chiarire" (Casula 1979: 77). L'esame di alcuni aspetti della vita sociale del giudicato conferma comunque la presenza di una marcata influenza toscana e genovese e l'adozione di moduli culturali provenienti dalla nostra penisola.

Nell'indagare il contesto sociale del giudicato di Arborea, Casula (1979: 80) fa notare come importanti cariche siano attribuite a personaggi appartenenti agli ambienti culturali dell'oltre Tirreno: l'arcivescovo di Oristano Guido Cattaneo è probabilmente, secondo Casula, un pisano; il notaio Giovanni Selle ed il medico dei giudici Grazia Orlandi provengono dai centri della nostra penisola. Altri aspetti dell'ambiente giudicale, e soprattutto dello scrittorio, riconducono ad una matrice non catalana: la carta impiegata, grigia con filigrana a vergelle, è diversa da quella usata dalla cancelleria catalana (cfr. Casula 1979: 81); ugualmente la scrittura delle carte arborensi riconduce per le sue caratteristiche ai moduli peninsulari. Non bisogna pensare, però, che questi tratti dello scrittorio giudicale possano essere attribuiti al ruolo dell'arcivescovo Guido Cattaneo, forse cancelliere del giudicato, perché sottolinea Casula (1979: 84) "L'appunto sarebbe valido se... non si notasse nello Scrittorio di 
Oristano una costante prelazione per i tipi della nostra penisola fino alla caduta del piccolo regno sardo, nel 1410".

Anche per quel che concerne la datazione il giudicato di Arborea si differenzia rispetto al regno di Aragona; nel giudicato sardo infatti è adottato lo stile dell'Incarnazione pisana, mentre nel regno di Aragona il computo del tempo avviene secondo lo stile dell'Incarnazione fiorentina.

Casula (1978 e 1979) rimarca in più punti "I'alterità" culturale del giudicato di Arborea rispetto all'ambiente dei dominatori; lo studioso esamina questo aspetto del giudicato sardo approfondendo lo studio della variegata composizione culturale che caratterizza il contesto arborense. I moduli che possono essere attribuiti all'influenza culturale della penisola vengono analizzati sia nel periodo che vede i regoli alleati degli aragonesi, sia nell'arco di tempo durante il quale gli arborensi si contrappongono all potere catalano. La datazione secondo lo stile pisano, per esempio, è adottata anche durante il periodo precedente la ribellione degli arborensi, per quanto il re aragonese, Pietro IV il Cerimonioso, avesse ordinato nel 1350 " che in tutti i territori della Corona (compresa la Sardegna), per evitare l'eterogeneità cronologica nelle scritture, si adottasse lo Stile della Natività (25 dicembre del 753 di Roma)" (Casula 1979: 88). L'alterità culturale, tuttavia, viene intesa anche come un atteggiamento di opposizione nei confronti dell'ambiente iberico: "è evidente che la diversità di lingua, di costumi, di mentalità e, forse, un atteggiamento di superiorità nei confronti dei Sardi da parte degli Aragonesi, mal accetto in generale e, in particolare, in un Paese che si considerava sovrano, fece sì che l'Arborea rimanesse sostanzialmente fedele alla tradizione italiana, ormai recepita da secoli ed adattata alle esigenze locali" (Casula 1979: 85). Persino l'esame delle opere attistiche dell'area arborense riconduce all' ambiente culturale della nostra penisola: il polittico di Ottana, per esempio, la cui esecuzione avviene presumibilmente fra il 1338 e il 1343, secondo i critici è opera di un pittore toscano o meridionale, influenzato comunque dalla cultura toscana: l'anonimo pittore potrebbe essere un giottesco pisano, un meridionale, verosimilmente un napoletano, che risente dell'influsso della cultura toscana oppure un artista dell' ambiente napoletano formatosi sotto l'influenza di Oderisi da Benevento (cfr. Casula 1979: 90).

Questa prevalenza della cultura proveniente dalla penisola non deve essere intesa, però, come una tendenza che mira ad escludere $o$, per lo meno, ad emarginare altre componenti culturali. Come rileva Maninchedda (1987: 19) riferendosi al condaghe di Santa Chiara, l'area arborense rappresenta un punto di incontro di più culture, dove diversi codici linguistici si alternano nei contesti comunicativi. Precedentemente abbiamo accennato al fatto che non solamente le interferenze linguistiche costituiscono per l'area arborense una testimonianza della compresenza di più sistemi linguistici, poiché l'interferenza è dovuta prevalentemente all'esposizione o alla pratica, anche relativa, che l'utente ha con due o più codici. Per l'area arborense disponiamo di documenti che testimoniano l'uso alternativo delle lingue da parte di una stessa persona. Le carte reali del giudicato di Arborea, pubblicate da Casula (1970 e 1977) e da D'Arienzo (1970), confermano la diffusione di più codici linguistici nella prassi scrit- 
toria. Fra le carte reali di Alfonso III il Benigno segnaliamo una lettera di Munillio Cicirera (?) e di Giovanni Uta diretta ad Ugone di Arborea, in data 7 febbraio 1331, che è redatta in sardo (cfr. Casula 1970: 114); il 9 febbraio 1331 Ugone di Arborea scrive in latino una lettera al governatore di Sardegna Raimondo de Cardona (cfr. Casula 1970: 114), mentre lo stesso Ugone riceve due lettere redatte in toscano, una da un anonimo l'11 gennaio (s.a.) (cfr. Casula 1970: 120) e l'altra da Francesco Satio, marito della sorella del regolo, in data 2 febbraio (s.a.) (cfr. Casula 1970: 201). Fra le carte reali di Giovanni il Cacciatore segnaliamo le lettere di Brancaleone Doria, marito della reggente Eleonora, redatte in catalano e in toscano; la prima lettera, datata 8 marzo 1393, è diretta al governatore del Capo di Logudoro Francesco Montbui ed è scritta in catalano, mentre la seconda lettera del 10 febbraio 1392 è diretta ad un nobile siciliano Andreotto Chiaramonte ed è redatta in toscano; quest'ultima lettera, come vedremo, è di particolare importanza in virtù di alcuni tratti dovuti all'interferenza di altre varietà linguistiche.

Le carte reali di Alfonso III il Benigno, di Pietro IV il Cerimonioso e di Giovanni I il Cacciatore forniscono utili informazioni sui contatti che $\mathrm{i}$ sardi, e non solo gli arborensi, hanno con gli ambienti dell'oltre Tirreno. Numerosi sono i documenti nei quali gli autori delle lettere, soprattutto catalani, manifestano le proprie preoccupazioni per le continue presenze pisane e genovesi. Il 13 marzo 1388, in seguito ai soprusi subiti dai genovesi ad opera di alcuni catalani, Giovanni I ricorda al governatore di Sardegna il patto tra Pietro il Cerimonioso e il comune di Genova che esclude simili atti (cfr. Casula 1977: 38-9). Il 31 (sic!) settembre 1391 Giovanni de Montbui, governatore dell'isola, i consiglieri ed i probiuomini di Cagliari inviano una lettera ad Antonio de Pujalt e Francesco Roig con la quale informano "sui movimenti bellici di Brancaleone Doria e sulla presa del castello di Sanluri da parte degli Arborea. Sempre i mittenti chiedono poi rinforzi per difendere il quartiere del porto di Cagliari e la villa di Alghero, e chiedono altresì un intervento del re presso i Comuni di Pisa e Genova affinché in virtù della pace stipulata cessino d'inviare vettovaglie e armi in Sardegna ai ribelli" (Casula 1977: 56-7). Abbiamo segnalato solamente due documenti che testimoniano la presenza, e l'ingerenza, dei pisani e dei genovesi nelle vicende della Sardegna ormai catalana, ma le carte reali aragonesi pubblicate da Casula (1970 e 1977) e da D'Arienzo (1970) forniscono un interessante quadro dei contatti fra i sardi e gli ambienti pisani e genovesi.

Durante il primo secolo della dominazione catalana in Sardegna, i rapporti militari, ma anche commerciali, dei sardi con gli operatori della nostra penisola non sono limitati agli ambienti pisani e genovesi. Il 12 novembre del 1334 il re Giacomo di Maiorca scrive ad Alfonso di Aragona informandolo che un'armata "composta da 700 cavalieri genovesi, milanesi, pisani e di altri Comuni italiani" (Casula 1970: 183) si accinge a partire per la Sardegna allo scopo di conquistarla; con la carta del 5 agosto 1341 Pietro IV il Cerimonioso ordina "al governatore generale, al vicario, ai baiuli, agli amministratori, ai doganieri e agli altri ufficiali dell'isola [...] di usare molte attenzioni nei confronti dei veneti che vengono in Sardegna con le loro merci" (D'A- 
rienzo 1970: 64). Durante il giudicato di Mariano IV, ricaviamo da Casula (1979: 90-1), quando ormai si ha la contrapposizione fra gli arborensi ed i catalani, viene data la caccia agli aragonesi; uno dei mezzi utilizzati per riconoscere un catalano è la verifica della competenza del sardo, ma purtroppo due giocolieri siciliani che si trovano a Bosa vengono uccisi perché, non dimostrando di conoscere il sardo, sono considerati aragonesi.

$\mathrm{NeI}$ XIV secolo l'ambiente arborense, e anche l'intera Sardegna, appare come un ambiente composito, nel quale operano componenti provenienti da diverse aree; certamente in molti casi si tratta di stanziamenti temporanei, di contatti anche commerciali saltuari che comunque presuppongono un non-isolamento della Sardegna rispetto alla realtà d'oltremare. L'isola, ormai iberizzata con l'affermazione degli aragonesi, continua ad avere contatti soprattutto con i pisani ed i genovesi non condizionati solamente dai possedimenti sardi delle nobili famiglie, mentre si assiste alle presenze sporadiche di operatori che provengono da altri punti della nostra penisola.

Il giudicato di Arborea è legato all'ambiente catalano; Mariano IV sposa la catalana Beatrice de Cabrera-Roccaberti; "Aveva trascorso parte della propria giovinezza in Catalogna, dov'era trattato come un principe; nel 1333 gli era stato conferito perfino il cingolo militare; l'11 settembre 1339 era stato creato, dal re d'Aragona, conte del Goceano" (Casula 1979: 88); ma questi contatti con l'ambiente aragonese non influenzano fondamentalmente il giudicato sardo, che mantiene i moduli soprattutto pisani.

Nell'esaminare le caratteristiche della cancelleria del giudicato di Arborea Casula (1978) rileva i tratti fondamentalmente pisani, sottolineando taluni aspetti che, durante il regno di Barisone I, riconducono ad una matrice genovese. La scrittura dell'Oristanese, afferma Casula, rimane quella gotica cancelleresca italiana; ma l'adesione ai moduli estranei alla cultura catalana è testimoniata da altre caratteristiche; la cultura del giudicato di Arborea è fondamentalmente composita, formata da tratti pisani, genovesi e catalani in un contesto sardo. Lo stesso Mariano IV usa la lingua catalana e toscana, oltre ovviamente al sardo; scrive in sardo oppure in toscano a seconda della lingua del destinatario; le stesse soldatesche del regolo, composte da toscani, tedeschi, inglesi, vengono assoldate a Pisa e alla loro guida sono Cicarello di Montepulciano, Giuliano di Massa, Meylun Birri, Mariano Sassone, oltre ai sardi Guantino de Serra e Cino de Sori (cfr. Casula 1978: 57 e 129).

Le differenti componenti culturali emergono chiaramente nella prassi scrittoria. Come si è detto, Mariano IV redige i propri ordini in sardo oppure in toscano; così scrive in una lettera riportata nei Procesos de Arborea, della quale citiamo un brano tratto dall'edizione di Casula (1978: 57):

"Ecco que due sclavus, unu gregu que at nomine lorgi et unu acteru niellu qui at nomine Nigola, sunt fuidus dae Castellu de Castru et sunt benidus in sas dictas terras nostras pro sos qualis vat so portadore de sa presente lictera nostra que llos conoscet. Et però vos mandamus que sos dictos sclavus in qualuncha logu et parti de sas terras 
nostras siant accatadus a peticione et requesta dessu portadore dessa presente los depiadis tenere et mandari suta bona guardia die nostru de presente que non venguant minus. Veramente per issa presente lictera et comandamentu non intendemus derogari nen rumpiri sos privilegios et immunitadis de cussos logos qui sunt privilegiadus et ant franquicies que talis hominis bogare non si 'nde potant, ma cussos privilegios et minitadis deppiant esser conservadus et conservadas in sa firmità issoro, sença mutacione alcuna."

Il testo presenta $i$ caratteri tipici dell'arborense: l'alternanza delle vocali finali $-i$ ed -e nelle forme verbali tenere, bogare, mandari, derogari, rumpiri, mentre negli esiti nominali ed aggettivali è costante l'impiego di -e: nomine, portadore, peticione, mutacione, presente; l'oscillazione nella desinenza del plurale -os ed -us in dictos, privilegios, logos, sclavus, fuidus, benidus, accatadus, privilegiadus, conservadus, con una marcata prevalenza del tipo in -us. Un dato rilevante del nostro testo è rappresentato dall'assenza della prostesi sia di $i$ - davanti a $s+$ consonante sia di $a$-dinanzi a $r$, fenomeno che invece è largamente attestato nei testi arborensi dei secoli XII-XVI. Il pronome atono di $3^{\circ}$ persona è rappresentato da los che compare scritto anche con la geminata llos, tratto diffuso negli altri documenti arborensi (cfr. Virdis 1982, XXIV).

Nel nostro testo ricorrono numerosi toscanismi, alcuni dei quali sono attestati negli antichi documenti sardi. A scopo esemplificativo riportiamo talune forme toscane: $E c c o$ è un toscanismo attestato nei condaghi di San Pietro di Silki (XII sec.) e di San Nicola di Trullas (XII-XIII sec.) (cfr. Wagner DES, s.v. akkò) ${ }^{8}$; qualis, qualuncha, che ricorre negli Statuti sassaresi (XIV sec.), nel condaghe di Santa Maria di Bonarcado e nella Carta de Logu nelle varianti qualunqua, calunqua, kalunka, calunca (cfr. Wagner DES, s.v. kále, -i); requesta, probabile toscanismo che compare anche nella Carta de Logu (cfr. Wagner DES, s.v. rekedere); guardia (cfr. Wagner DES, s.v. gwardare); nomine che risente della forma toscana in relazione all'esito della vocale tonica (cfr. Wagner DES, s.v. númene); portadore (cfr. Wagner DES, s.v. portare, -ai); però; veramente; privilegios; privilegiados; firmità; sença, un toscanismo presente anche negli Statuti di Sassari nella variante senza e nella Carta de Logu de Arborea, nella quale le forme sono costantemente senza e sença (cfr. Wagner DES, s.v. sene). Citiamo infine franquicies come forma che potrebbe far pensare ad un influsso iberico (cfr. Wagner DES, s.v. fránku).

7 "Da poco due schiavi, uno greco che ha nome Giorgio e un altro nero che ha nome Nicola, sono fuggiti da Castello di Castro e sono venuti nelle dette terre nostre, per i quali va il latore della presente nostra lettera che li conosce. E perciò vi ordiniamo che i detti schiavi in qualunque luogo e parte delle terre nostre siano trovati, a domanda e richiesta del latore della presente li dobbiate arrestare e mandare sotto buona sorveglianza così che, al momento, non vengano meno. In verità con la presente lettera e ordine non intendiamo derogare né contravvenirc ai privilegi e alle immunità di quei luoghi che sono privilegiati e hanno franchigie per cui tali uomini non ne possano essere chiamati in giudizio, ma quei privilegi e immunità debbano essere conservati e conservate nella loro legittimità, senza che vengono mutati".

8 Per l'esito que rinviamo a Sanna (1975: 136). 
I prestiti che abbiamo riportato, alcuni dei quali non sono registrati dal Wagner, possono essere considerati come forme ormai inserite nelle varietà sarde; Mariano IV, tuttavia, nella prassi scrittoria impiega il toscano del quale ha una certa competenza; così scrive il regolo:

"Et se compratore altro aver non ne potrano, che la corte lo comprarà; et chiuncha vendere non lo vorrà, lo potrà avere ad ogna loro voluntà e piacere, cussì da Arestano come da Murreali, dechiarando ad ciascuno que questo si fa per que, se li predicti venissino forçatamente, che resistencia non si potesse avere, over, essendo la gente nostra in alcuna parte che succurrere acconciamente non si potesse, andando scurrendo per quelle parte, non possano trovare nen grano nen panaticha nen reffreschamento e victualia alcuna; e non trovando victualia nen grano, lo dito stolo non potrà stare, $\mathrm{e}$ conviene che partire si debbia, per che elli non anno victualia, nen da Castello avere non potrano."

Nella lettera di Mariano IV ricorrono alcune forme rilevanti che riportiamo, riferendoci non solamente al brano riprodotto ma all'intero testo: luocho con il dittongamento tipico pisano (ma la forma non è sonorizzata) contro stolo; parte (in nele parte) con la formazione del plurale in -e, presente nel pisano ma anche nelle varietà di altre aree toscane; tra le forme verbali notevoli segnaliamo serano e venissino, esito pisano con la formazione della terza persona plurale dell'imperfetto congiuntivo in - $n$-. Altri tratti del nostro testo rinviano all'area toscana occidentale quali l'occorrenza di ogna (ma compare anche ogni), l'impiego del tipo preposizionale in nel (in nel campo, in nele parte de Kallari), la presenza del dittongo au in paraula, la sonorizzazione in seguro contro secundo. Segnaliamo inoltre la modificazione di $o$ in $u$ in cussi e l'occorrenza di predicti, dito tipica, come è noto, del pisano e del lucchese (cfr. Castellani 1952, 1965, 1974 e Dardano 1967); si rileva infine l'assenza dell'assimilazione del gruppo consonantico - ct- in victualia e in predicti contro dito con la scempia.

Casula (1979: 95-6) indaga l'ambiente culturale nel quale vive Mariano IV; circondato da persone istruite quali i giurisperiti Filippo Mameli, fra' Leoni di Ravenna, Bartolo Catone, Guido de Vada, i medici Grazia Orlandi, Maestro Giacomo e Maestro Corardo, "anche Mariano IV doveva essere colto e raffinato. Sapeva [...] il latino, l'italiano, il sardo e il catalano; era in corrispondenza epistolare con la giovane Caterina da Siena; conosceva l'astrologia e le teorie di Tolomeo; aveva sempre a portata di mano una Bibbia" (Casula 1979: 96-7). Tuttavia a prescindere dalla lingua impiegata nella scrittura, rileva sempre Casula (1978: 59), Mariano IV usa il formulario della cancelleria regia aragonese; lo studioso individua i tratti catalani sia negli ordini di esecuzione sia nelle comunicazioni dirette ai sudditi. Anche alcune caratteristiche esteme dei documenti richiamano il modello aragonese: "gli scritti "chiusi" erano sigillati, in genere, col sigillo segreto; quando, invece, erano "aperti" avevano il sigillo maggiore, che in Catalogna si chiamava sigillo della maestà. Entrambi erano sigilli "aderenti" di autenticazione, solo in speciali casi usati anche per chiudere il documen-

Cit. da Casula (1978: 58-9). 
to e rendere riservato il contenuto" (Casula 1978: 59). La cancelleria arborense si presenta composita, con caratteristiche tipiche catalane e con peculiarità invece peninsulari, le prime limitate all'apparato di produzione dei documenti e alla loro sigillatura, le seconde riguardanti l'aspetto culturale documentario e derivanti soprattutto dall'assorbimento dei modelli pisani e genovesi; il tipo di scrittura, l'assenza della nota marginale, la disposizione delle righe sono tratti che avvicinano lo scrittorio arborense al modello peninsulare. Come si è detto precedentemente, la scrittura è la minuscola cancelleresca italiana, secondo la definizione di Casula (1978: 83), importata probabilmente da religiosi; tanto la formulazione del documento quanto la sua stesura sono affidate, sottolinea Casula, a persone dotate di una cultura che richiama la nostra penisola. La mancanza della nota dorsale, rileva Casula, è caratteristica anche dei documenti prodotti da altre cancellerie peninsulari; ugualmente la disposizione del rigo di scrittura non è parallelo al lato maggiore, ma segue "il lato minore, come si faceva più a Pisa che in Catalogna" (Casula 1978: 80). In seguito, con Brancaleone Doria, lo scrittorio arborense avrà taluni tratti genovesi.

Anche con Ugone III, succeduto al padre Mariano IV nel 1375, si ha un persistere dell'ambiente culturale che conduce alle città dell'oltre Tirreno, con l'accentuazione però di un atteggiamento anticatalano; ma è nel periodo durante il quale il giudicato è retto da Eleonora di Arborea che si nota un'ulteriore influenza dei modelli peninsulari sull'ambiente giudicale. La presenza nel giudicato di Brancaleone Doria, marito della reggente, contribuisce a rafforzare infatti la componente genovese nell'area arborense.

Brancaleone Doria, afferma Casula (1979: 104), conosce il sardo, il toscano, il latino e il catalano. Il 10 febbraio 1392 invia al nobile siciliano Andreotto Chiaromonte una lettera, redatta in toscano, della quale riportiamo un brano secondo l'edizione di Casula (1979: 106).

"Et questo sarà in voluntà de la potentia divina, sigondo che la sua gratia volrà administrare; et lantora non vi calirà più dubitare dilloro, et che seguramente porrete stare in vostra Casa et prospero stamento. Et se per aventura la dicta armata [terrà dirita] via cotesta [isula] sensa venire di qua, piacciavi, caro frate, che la vostra vertù con operi valorosi et maneri laudabili vi sia efficaci in [loro] dampagio et crudele destructione. Ma piacesse a lo omnipotente Dio che per sua gratia et misericordia ni consentisse che da questa isula di Sardigna a cotesta isula di Cicilia havesse un ponte che per terra si potesse passare, perchè lantora volunterimente vi soccorririamo ala dicta armata, et noy personivilmente ni troveriemo insieme con voy con cinqui milia pedoni sardi et mille sardi da cavallo almeno, inperciò che con la vostra posansa insieme, et con lo vostro senno et virtù, sensa dubio li dicti iniqui et malvagi Cathalani meteriamo ad morte et ad crudelissimo destrugimento, et la loro superbia et presumptuosa arrogantia conculchariamo, in manera che sariamo pagati di tutti loro traitioni et malvastai che anno usati in ver di noy. Et voy, come ditto havemo di sopra, staresti a segurtà sensa paura dilloro." 
Fra i tratti rilevanti che riguardano le consonanti segnaliamo la sonorizzazione in sigondo, seguramente, segurtà, l'occorrenza di $s$ al posto di $z$-caratteristica tipica del pisano e del lucchese- in sensa, posansa, l'assenza dell'assimilazione, in seguito alla sincope vocalica precedente $r$, in volrà, il rafforzamento sintattico iniziale in dilloro. Per quel che concerne il vocalismo notiamo invece le forme pisano-lucchesi dicta, dicti, ditto, il mantenimento del dittongo in laudabili, il dittongamento in troveriemo, l'occorrenza di $u$ in luogo di $o$ in voluntà, volunterimente. Segnaliamo infine la forma notevole funno e l'impiego del suffisso-ivil- in personivilmente ${ }^{10}$.

In questo brano che abbiamo riportato occorre la forma lessicale lantora "allora" in "et lantora non vi calirà più dubitare dilloro" e in "perché lantora volunterimente vi soccorririamo ala dicta armata"; si tratta di un prestito genovese (dall'antico genovese lantór, lantora; cfr. Wagner DES, s.v.) che negli antichi documenti sardi ricorre, sempre secondo il Wagner, solamente negli Statuti di Castelsardo. Brancaleone Doria, come è noto, è di origine genovese e la sua cultura, rileva Casula (1979: 103) è di tipo ligure; sono dovute pertanto all'interferenza del genovese, varietà compresente con il toscano nel repertorio linguistico del Doria, le occorrenze di lantora. Persino lo scrittorio di Casteldoria di Brancaleone segue il modello, afferma Casula (1978 e 1979), del comune di Genova; ma con l'insediamento del Doria nel giudicato di Arborea $\mathbf{i}$ tratti genovesi si fondono con quelli pisani e sardi.

Oltre che il toscano e, come vedremo, il sardo anche il catalano è il codice che viene utilizzato nella redazione delle lettere di Brancaleone Doria. L'8 marzo 1393 Brancaleone Doria scrive a Francesco de Montbui e la lingua impiegata è il catalano (cfr. Casula 1977: 79-80); ugualmente, è redatta in catalano la lettera (s. d.) diretta a Giovanni de Montbui, governatore di Sardegna (cfr. Casula 1977: 185-9), nella quale il Doria rimarca le origini storiche, autonome del giudicato di Arborea.

In altri documenti il Doria dimostra di possedere una buona conoscenza del sardo; così scrive il 10 luglio 1391 in un "mandato", del quale riportiamo un brano secondo l'edizione di Casula (1979: 104):

"In tanto que sos ambaxadoris proprios qui si mandant in Cadalongia dae Cicilia per issu comte de Piralta et atteros seguacis suos sont istados in sa citadi nostra de Aristani, arrivados per fortuna de tempus, et nos ant contadu per ordine tottu su fattu, et qui in ogni modu sa armada si istabiliscit et mittet se in punta de passare a tottu podere, pro ssa quale causa, considerando vos sa suspecione qui iay in sa predicta letra nostra declarato vos amus, et dubitando qui su governadore de sa Lighera su quali sendu qui semus certo quirchat de frunire et istabilire cussu castellu de cussa citade, non vos illos condugat a deberlu frunire et istabilire cussu castellu ad medu suo cum inganni et vitiu, cum sa presente letra nostra vos faghimus avistos et pro utile et bene vostru cum salvamento, et per consequente de tottu sa nacione sardischa, vos consi-

10 Per la caratterizzazione di questi esiti diffusi in differenti aree toscane rinviamo a Castellani (1952, 1965, 1974) e a Dardano (1967). 
giamus et mandamus qui in neuna manera non depiades consentire nen sufferre qui su castellu fruniat et non istabilischat de vituagia nen de cosa alcuna."11

Ad eccezione dell'esito -is in ambaxadoris e in seguacis, in questo testo si rivela una netta prevalenza dei tratti che l'arborense condivide con il logudorese: la terminazione -os per la formazione del plurale (proprios, atteros, istados, arrivados, avistos contro l'unica occorrenza di - $u$ s in tempus); l'esito della vocale finale - $e$ nelle forme nominali e aggettivali (ordine, suspecione, governadore, citade, bene, nacione, utile, presente contro $-i$ in citadi); la terminazione verbale costantemente in -e (passare, frunire, istabilire, consentire, sufferre).

Fra i numerosi toscanismi presenti nel brano riportato citiamo le seguenti forme, alcune delle quali non sono registrate dal Wagner: in tanto que (cfr. Wagner DES, s.v. intántu), arrivados (cfr. Wagner DES, s.v. arrivare), ogni (cfr. Wagner DES, s.v. onnia), fattu (con il significato di "evento" già segnalato da Maninchedda 1987), módu (cfr. Wagner DES, s.v.), quale, quali (cfr. Wagner DES, s.v. kále, -i), istabiliscit, istabilire e istabilischat (cfr. Wagner DES, s.v. istabilire), dubitando (cfr. Wagner DES, S.v. dudare), certo (cfr. Wagner DES, s.v. čértu), avistos, nacione (cfr. Wagner DES, S.v. natsiòne), neuna (cfr. Wagner DES, s.v. niunu), vituagia che ricorre anche nello Statuto di Castelsardo e nel codice di Sorres (Wagner DES, s.v. vittual'a rifiuta l'interpretazione secondo la quale la forma vituagia sarebbe un genovesismo), cosa (cfr. Wagner DES, s.v. kòsa), alcuna (cfr. Wagner DES, s.v. alicunu), ecc. A proposito di frunire e di fruniat Wagner (DES, s.v. frunire) segnala la corrispondenza della voce sarda con le forme dell'italiano fornire e del catalano fornir. Abbiamo citato solo alcuni toscanismi, ma nella lettera di Brancaleone Doria si rilevano numerosi esiti dovuti al contatto del sardo con il toscano.

La compresenza di più varietà nel repertorio linguistico di Brancaleone Doria è dovuta certamente ai rapporti che il nobile genovese intrattiene con gli ambienti peninsulari. Spesso si tratta di rapporti di tipo stategico, miranti ad instaurare un contatto con forze che possono agevolare i disegni espansionistici del Doria ai danni degli aragonesi. La stessa lettera inviata ad Andreotto Chiaramonte, della quale abbiamo riportato un brano, ha lo scopo di esortare il nobile siciliano ad opporsi ai catalani che si accingono a sbarcare in Sicilia; il 12 marzo 1393 il governatore di Sardegna comunica al re aragonese che $\mathrm{i}$ genovesi "non rispettano la pace, danneggiano l'isola $\mathrm{e}$ armano navi a Bonifacio per assalire legni catalani con atti di pirateria, e come rinfor-

11 "dal momento che gli ambasciatori particolari inviati in Catalogna dalla Sicilia ad opera del conte di Piralta ed altri suoi seguaci sono stati nella nostra città di Oristano, (ivi) arrivati per circostanze legate alle condizioni del tempo, e ci hanno raccontato ordinatamente tutto il fatto, e che in ogni modo l'armata si va rafforzando e sta per raggiungere la piena operatività; per la qual causa, tenendo conto del sospetto che vi abbiamo già dichiarato nella predetta lettera, e temendo che il governatore di Alghero, il quale cerca di rifornire e di rafforzare il castello di codesta città, vi induca a rifornire e a rafforzare tale castello a modo suo, con l'inganno e l'imbroglio, per mezzo della presente lettera vi rendiamo avvertiti e per il vostro utile, per il bene vostro e per la vostra salvezza, e quindi di tutta la nazione sarda, vi consigliamo e vi ordiniamo che in nessuna maniera dobbiate consentire e tollerare che rifornisca il castello e lo provveda di vettovagliamento, né di alcuna cosa". 
zano il potenziale bellico di Brancaleone Doria il quale avrebbe in mente di occupare Cagliari con uno stratagemma" (Casula 1977: 83). Le azioni del nobile sardo-genovese e l'occupazione del castello di Sanluri da parte degli Arborea costituiscono un pericolo, tanto che Giovanni de Montbui informa alcuni nobili sugli avvenimenti con la lettera datata settembre 1391, della quale si è parlato precedentemente (cfr. Casula 1977: 56-7); il 12 agosto 1391 Giovanni Castello informa il catalano Lorens Loques sui tentativi di Brancaleone Doria di occupare Alghero (cfr. Casula 1977: 50-5). Le mire espansionistiche del Doria preoccupano i catalani e richiedono l'impegno di ingenti forze aragonesi e dello stesso re Giovanni I per contrastare le azioni degli arborensi (cfr. Casula 1977: 88); il 5 febbraio (s.a.) i "consiglieri e i probiuomini del Castello di Cagliari scrivono all'infante Martino, duca di Montblanc, per metterlo al corrente sulla situazione militare dell'isola e della necessità di un intervento armato del re d'Aragona per fermare Brancaleone Doria" (Casula 1977: 122).

I continui contatti del Doria con gli ambienti d'oltremare comportano certamente l'assorbimento dei moduli peninsulari; si tratta di rapporti di tipo politico allo scopo di assicurare all'Arborea l'alleanza con altre forze, accomunate dall'atteggiamento anticatalano, e al fine soprattutto di reclutare soldatesche per contrastare gli aragonesi. L'assorbimento dei moduli peninsulari, però, è dovuto in modo particolare all'ambiente d'origine del nobile, quello ligure, e al contesto composito, per quel che concerne la componente culturale, del giudicato di Arborea nel quale Brancaleone Doria ormai opera.

L'Arborea, come si è visto, rimane sempre legata agli ambienti delle città dell'oltre Tirreno; nell'esaminare i manoscritti arborensi contenenti notazioni musicali Milia (1987: 215-7) sottolinea i legami che intercorrono fra l'area oristanese e gli ambienti culturali della penisola, legami che sono attestati negli antichi documenti del XII-XIII secolo e del XIV secolo. La prima attestazione è contenuta in "quattro frammenti pergamenacei [...] conservati nella Cattedrale di Oristano, dove furono utilizzati come materiale di recupero per la rilegatura di alcuni più tardi codici liturgico-musicali. I suoi neumi, disposti su un tetragramma con una linea rossa indicante il $f a$, ci riportano ad un tipo di notazione neumatica particolarmente in uso nell'Italia meridionale, fino a Roma, che viene definita beneventana (Milia 1987: 216). La seconda attestazione del XIV secolo, più significativa perché prodotta in un contesto politico catalano, è un codice conservato nell'archivio del monastero oristanese di Santa Chiara e che contiene, oltre alla regola delle monache, "antifone e salmi in notazione musicale su tetragramma“ (Milia 1987: 218).

Il manoscritto, che è stato rinvenuto e pubblicato da Mele (1985), rappresenta un importante documento per la conoscenza della variegata composizione culturale del giudicato di Arborea. Scritto "in una gotica libraria [...] orientata verso quella semplificazione $[. .$.$] che caratterizza la littera textualis italiana della seconda metà del "300"$ (D’Arienzo 1985: 7), il codice oristanese fornisce numerose informazioni sulla vita del giudicato arborense caratterizzato, è stato affermato, da una civiltà autoctona ${ }^{12}$.

12 Cfr. D’Arienzo (1985). 
Nella sua interessante analisi sulle caratteristiche della musica arborense nel Trecento, Mele (1984) indaga i tratti relativi ai cerimoniali di corte e riconducibili all'ambiente catalano-aragonese e queste peculiarità vengono ribadite da Mele (1985: 34): alla "corte di Oristano, all'epoca di Mariano IV, l'inizio e la fine dei pasti del giudice erano sottolineati dall'intervento di diversi strumentisti [...]. Nel cerimoniale arborense è evidente un forte influsso catalano-aragonese, poiché nelle Ordinanze di Corte di Pietro IV il Cerimonioso (1344) si prescrive che quattro juglars (due trompadors, un tabaler e un trompeta) erano tenuti ad annunciare appunto l'inizio e la fine dei pasti reali".

Il codice oristanese però, che risale al periodo in cui il giudicato è retto da Mariano IV, Ugone III e da Eleonora, rivela delle caratteristiche che riconducono anche all'ambiente culturale della penisola. Oltre alla scrittura, i cui tratti salienti sono rappresentati dai moduli in uso nelle cancellerie penisulari, alcuni aspetti del tipo di notazione musicale, talune informazioni sulla vita monastica risentono dell'influsso dei modelli provenienti dall'oltre Tirreno; a proposito della pratica dei salassi, per esempio, D'Arienzo (1985: 9) sottolinea la differenza che intercorre fra la prescrizione prevista per le clarisse oristanesi, che possono ricorrere al salasso non più di tre volte all'anno, e la norma riguardante le monache parigine alle quali è consentita la pratica del salasso per sei volte all'anno.

Il codice di Oristano ha una notevole importanza sotto molteplici aspetti, non ultimo il fatto che si tratta di uno dei pochi codici coevi alla fondazione del monastero (cfr. D'Arienzo 1985: 9). Anche la lingua del documento si rivela di particolare interesse poiché, afferma Mele (1985: 18), è redatto "in un latino tipicamente medievale e scorretto, nel quale talvolta sono presenti fenomeni di interferenza tra la fonetica propria del copista e il testo latino"; nella c. 32 r, per quanto aggiunta da una mano più tarda, compare la scritta in sardo "pro sa benedicioni dessu ora(cio) Domine lesu Christe in su missali et pustis sas siguitivas". Sarebbe auspicabile uno studio particolareggiato delle caratteristiche linguistiche di questo documento prodotto in un'area linguisticamente composita e che, nella prassi scrittoria, risente dell' influenza di più codici. La pregevole edizione di Mele, che opportunamente ha fornito "una trascrizione fedele del testimone arborense tesa a restituire la lezione dello scriba in tutta la sua integrità, anche quando si presenta sotto forme astruse e scorrette" (Mele 1985: 41), consente uno studio approfondito delle peculiarità, anche grafiche, del latino medievale in Sardegna, proprio in virtù della fedele riproduzione del codice priva degli interventi del curatore che, nelle edizioni critiche, mirano e rendere più accessibile il testo; questi interventi, però, sottraggono e modificano un dato importante per un'indagine storica che voglia esaminare l'evoluzione delle forme grafiche.

Casula (1978: 82-9) indaga le particolarità della grafia adottata nel giudicato di Arborea allo scopo di fornire un quadro dei tratti che caratterizzano la scrittura cancelleresca arborense e che consentono di contrapporre la pratica scrittoria giudicale a quella catalana. Basandosi sull'esame dei documenti orginari della curia del giudicato, documenti che coprono un arco di tempo compreso fra il 1297 e il 1410, Casula 
mette in evidenza la specificità dei documenti della curia di Oristano, "la quale ci sembra facesse qualcosa di più che abbandonarsi all' esecuzione passiva e sciatta della grafia gotica appresa in Italia o importata dagli italiani, verosimilmente dai Pisani: $i$ Sardi oristanesi, infatti, calligrafarono questa scrittura fino alla fine del giudicato. In poche parole: con essa crearono la propria cancelleresca, che dopo il 1323 può essere contrapposta alla cancelleresca catalana delle scrivanie regie dell'isola" (Casula 1978: 83).

La matrice della gotica cancelleresca arborense sarebbe la minuscola cancelleresca italiana, la cui essenza, secondo Casula, è costituita dalla minuscola dei documenti pontifici. I tratti individuati da Casula sono elementi costanti presenti nei documenti dei giudici Ugone II, Pietro II, Mariano IV ed Eleonora, rappresentati da un tracciato acuto nei vertici delle lettere $i, m, n, t, u$ e dai caratteristici terminali delle lettere gambate $f, p, q$, s. Nonostante l'arco di tempo che separa la stesura del primo e dell'ultimo dei documenti esaminati da Casula, la scrittura "è sempre la stessa, tracciata in maniera tale che supera ed annulla i personalismi o "ductus" dei diversi scrivani [...], venendo a formare una scrittura caratteristica, facilmente riconoscibile, rappresentativa della cancelleria giudicale ed alla quale si può attribuire senz'altro il nome di cancelleresca arborense" (Casula 1978: 84).

Nell'esaminare i moduli delle cancellerie sarde lo studioso sottolinea l'eterogeneità di taluni documenti, derivante dall'ambiente composito culturalmente ed influenzato appunto da molteplici fattori, quali la circolazione dei notai e il ruolo dell'amanuense: in Casula (1978), per esempio, è trascritta una pergamena datata 5 febbraio 1326, di particolare interesse per la presenza di più tipi di scritture. Si tratta di una richiesta tendente ad ottenere la conferma delle immunità e delle franchigie previste per coloro che lavorano nelle saline del Cagliaritano, vantaggi che sono stati annullati dall'infeudazione della villa di Cepolla a Raimondo Ça Vall. Come rileva Casula (1978: 87) la pergamena ha la "completio" di un notaio proveniente dalla penisola, redatta secondo i tipi della gotica documentaria italiana, mentre il documento è scritto con i tipi della gotica cancelleresca catalana da un amanuense catalano e rappresenta, nota sempre Casula, l'espressione giuridica di una comunità sarda.

Il giudicato di Arborea costituisce solo un tassello nella variegata composizione isolana, rappresentativo di un'area linguistica, quella sarda, nella quale i differenti influssi culturali si intersecano dando luogo ad un multiforme assetto linguistico. Tanto più significativa appare l'alternanza delle lingue ed i fenomeni di interferenza che si manifestano nella prassi scrittoria se consideriamo il periodo al quale i documenti dell'ambiente arborense si riferiscono: si tratta di un arco di tempo che sotto il profilo politico vede l'Arborea, e la Sardegna, ormai legate all'ambiente iberico del quale vengono assorbiti taluni moduli; ma questi moduli coesistono con la tradizione sarda e con quella proveniente dalla penisola, tanto che persino in un documento più tardo, il condaghe di S. Chiara di Oristano, è possibile rilevare il coesistere della lingua sarda, toscana e catalana. Non si può "escludere che anche gli scriventi del XV-XVI 
secolo facciano riferimento ad una tradizione scritta sarda, in cui l'influenza della penisola italiana era già notevole" (Maninchedda 1987: 20).

\section{BIBLIOGRAFIA}

Anatra, B., Dall'unificazione aragonese ai Savoia, in Storia d'Italia, vol. X, Torino (UTET), 1984, pp. 189-654.

Artizzu, F., Pisani e Catalani nella Sardegna medioevale, Padova (CEDAM), 1973.

Artizzu, F., La Sardegna pisana e genovese, Sassari (Chiarella), 1985.

Atzori, M.T. (a cura di), Brogliaccio del Convento di S. Martino di Oristano, Parma (Scuola Tipografica Benedettina), 1956.

Atzori, M.T. (a cura di), Il Condaxi Cabrevadu, Modena (Società Tipografica Editrice Modenese), 1957.

Besta, E., Intorno ad alcune pergamene arborensi del secolo decimosecondo, in "Archivio Storico Sardo", 2 (1906), pp. 423-433.

Blancard, M. - Wescher, K., Charte sarde de l'abbaye de Saint-Victor de Marseille écrite en caractères grecs, in "Bibliothèque de l'Ecole des Chartes" 35 (1874), pp. 255-265.

Castellani, A., Nuovi testi fiorentini del Dugento, Firenze (Sansoni), 1952.

Castellani, A., Pisano e lucchese, 1965, in Castellani 1980: I., pp. 283-326.

Castellani, A., Lingua parlata e lingua scritta nella Toscana medievale, 1974, in Castellani 1980: I., pp. 36-48.

Castellani, A., Saggi di linguistica e filologia italiana e romanza, 3 voll., Roma (Salerno Editrice), 1980.

Casula, F.C., Carte reali diplomatiche di Alfonso III il Benigno, re d'Aragona, riguardanti l'Italia, Padova (CEDAM), 1970.

Casula, F.C., Carte reali diplomatiche di Giovanni I il Cacciatore, re d'Aragona, riguardanti l'Italia, Padova (CEDAM), 1977.

Casula, F.C., Breve storia della scrittura in Sardegna, Cagliari (EDES), 1978.

Casula, F.C., Cultura e scrittura nell'Arborea al tempo della Carta de Logu, in Il mondo della Carta de Logu, Cagliari (3 T), 1979, pp. 71-109.

Dardano, M., Note sul Bestiario toscano, in "L'Italia Dialettale" 30 (1967), pp. 31117.

D’Arienzo, L., I possessi catalani dei giudici d'Arborea, in "Studi Sardi” 21 (1968), pp. 134-146.

D'Arienzo, L., Carte reali diplomatiche di Pietro IV il Cerimonioso, re d'Aragona, riguardanti l'Italia, Padova (CEDAM), 1970.

D'Arienzo, L., Introduzione a Mele (1985), pp. 7-11. 
Guamerio, P.E. 1905, in Besta, E. - Guarnerio, P. E. (a cura di), Carta de Logu de Arborea. Testo con prefazioni illustrative, in "Studi Sassaresi" 3 (1905), pp. 1141+1-72.

Lazzeri, G., Antologia dei primi secoli della letteratura italiana, Milano (Hoepli), 1942, ristampa del 1954.

Maninchedda, P., Il Condaghe di Santa Chiara, Oristano (S'Alvure), 1987.

Maninchedda, P., Il sardo arborense nel Condaghe di S. Chiara, in "Biblioteca Francescana Sarda" 1 (1987 a), pp. 365-391.

Mele, G., La musica catalana nella Sardegna Medievale, in J. Carbonell - F.

Manconi, I catalani in Sardegna, Milano (Silvana Editoriale), 1984, pp. 187-192.

Mele, G., Un manoscritto arborense inedito del Trecento. Il codice 1 bR del monastero di Santa Chiara di Oristano, Oristano (S'Alvure), 1985.

Merci, P., Il più antico documento volgare arborense, in "Medioevo Romanzo" 5, pp. 362-383.

Milia, G., La civiltà giudicale, in AAVV, Storia dei sardi e della Sardegna, vol II: Il Medioevo dai giudicati agli aragonesi, Milano (Jaca Book), 1987, pp. 193-229.

Monteverdi, A. (a cura di), Testi volgari italiani anteriori al Duecento, Roma (Maglione), 1935.

Sanna, A., Il dialetto di Sassari (e altri saggi), Cagliari (Trois), 1975.

Solmi, A., Le Carte volgari dell'Archivio Arcivescovile di Cagliari, in "Archivio Storico Italiano", 5, pp. 273-330 e 3-65.

Virdis, M., Note sui dialetti dell'area arborense e la lingua del Condaghe di Santa Maria di Bonarcado, in Il Condaghe di S. Maria di Bonarcado, ristampa del testo a cura di E. Besta, Oristano (S'Alvure), 1982, pp. XXIII-XXXIX.

Virdis, M., Areallinguistik, in Holtus, G. - Metzeltin, M. - Schmitt, C. (a cura di), Lexikon der Romanistischen Linguistik, Band IV: Italienisch, Korsisch, Sardisch, Tübingen (Niemeyer), 1988, pp. 897-913.

Volpicella, L., Una carta di Arborea e Ponzio Pilato, in "Atti della Società ligure di Storia Patria " 53 (1926), pp. 63-90.

Wagner, M.L., La lingua sarda. Storia, spirito e forma, Bern (Francke Verlag), 1951.

Wagner, M.L., Dizionario etimologico sardo, Heidelberg (Winter), 1960-64. 


\section{JEZIK IN KULTURA PISARJEV V 14. STOLETJU: SODNA PISANJA V ARBOREI (SARDINIJA)}

V poznem srednjem veku raba latinščine popušča, vsaj za dokumente manj tehtne vsebine. Tudi na Sardiniji je to čas vzcveta ljudskega jezika, sardščine. 14. stoletje pomeni kritično obdobje: otoku vladajo sodniki, ob katalonščini (Aragonska krona je formalni gospodar otoka) je sarďčina še do konca stoletja deloma jezik uprave. Toskanski vpliv je zaznaven, tudi zaradi ekonomskega pritiska Pise. Avtorica analizira jezik notarjev in pisarjev na sodnikovem dvoru v Arborei, vseskozi pa tehta vpliv toskanizmov. Ti so včasih samo grafični, včasih morfološki, vidni zlasti pri morfemih: sardščina, npr., je iz latinščine ohranila končni $-s$, italijanščina in tako tudi toskanščina, pa ne; $v$ dokumentih so vidna oklevanja. Pojavljajo se tudi sardščini sicer znane besede, vendar v pomenu, ki je bil v Toskani domač, sardščina tiste dobe pa jih v takem pomenu ni poznala. 